\title{
Execution Scheme for Driver Drowsiness Detection using Yawning Feature
}

\author{
Monali V. Rajput \\ Vidyalankar Institute of Technology \\ Mumbai, India
}

\author{
J. W. Bakal, PhD. \\ Shivajirao S. Jondhale College of Engineering \\ Mumbai, India
}

\begin{abstract}
Fatigue and drowsiness of driver are amongst the most significant cause of road accidents. The main aim of the project is to find out the methods to detect driver drowsiness and alerting them hence increasing the transportation safety. By using many body and face gestures as a sign of driver fatigue, detection including yawning, eye tiredness, and eye movement, these condition shows that driver is not in proper driving condition.

In this proposed system yawning and eye movement are used. Driver fatigue can increase the chances of car accidents. The reason for this type of car accidents is due to the fact that driver fails to take necessary actions prior to the collision occurs, therefore assisting system which will monitor the behavior of driver and also will give the necessary alerts to the driver which will prevent the road accidents.
\end{abstract}

\section{Keywords}

Skin Segmentation, Template Matching, Eye Map, Mouth Map, Yawning Detection

\section{INTRODUCTION}

The advance of computing technology has provided the means for building intelligent vehicle systems. Drowsy driver detection system is one of the potential applications of intelligent vehicle systems. Excessive sleepiness results in an increased risk of motor vehicle crashes primarily because the driver either falls asleep while driving or experiences reduced attention to road events and the driving task due to fatigue. Statistics show that $20 \%$ of all the traffic accidents are due to drivers with a diminished vigilance level [2]. Furthermore, accidents related to driver hypo-vigilance are more serious than other types of accidents, since sleepy drivers often do not take correct action prior to a collision. For this reason, developing systems for monitoring driver's level of vigilance and alerting the driver, when he is drowsy and not paying adequate attention to the road, is essential to prevent accidents. The prevention of such accidents is a major focus of effort in the field of active safety research.

People in fatigue show some visual behaviors easily observable from changes in their facial features like eyes, head, mouth and face. Computer vision can be a natural and non-intrusive technique to monitor driver's vigilance. Faces are the important part to be considered while giving any judgment regarding drowsiness which has been a research target in computer vision for a long time. Automatic recognition of facial expression consists of three levels of tasks: face detection, facial expression information extraction, and expression classification. It involves detection, identification and tracking facial feature points under different illuminations, face orientations and facial expressions. The advantage of computer vision techniques is that they are non- invasive, and thus are more open to use by the common public. The critical issue that a fatigue detection system must address is the question of how to accurately and early detect fatigue at the initial stage. Possible non-intrusive techniques for detecting fatigue in drivers using computer vision are, based on eye and eyelid movements, based on head movement, methods based on mouth opening. In proposed method, the driver's face is taken as an input from camera. We can then detect the location of the eyes and the mouth in the detected face. The mouth geometrical features are then used to detect the yawn. Then system will generate the alert sound to the driver about his drowsiness and the improper driving state in case of yawning is detected [1].

\section{SYSTEM DESIGN}

The driver drowsiness behavior detection using yawning feature system consists of different module to properly analyze changes in the mouth of driver. These modules are categorized as,
a) Face Detection
b) Eye and Mouth Detection
d) Yawning Detection

\subsection{Face Detection}

Detecting faces is a crucial step and usually the first one in these identification applications. Most face detection systems attempt to extract a fraction of the whole face, thereby eliminating most of the background and other areas of an individual's head such as hair that are not necessary for the face recognition task. Different techniques are used for face detection like Knowledge-based methods, Feature invariant approaches, Appearance-based methods, Template matching method [11]. Here in this proposed system template matching method is used where several standard patterns stored to describe the face as a whole. Here we are not trying to classify an image as a 'face' or 'non-face' but are trying to recognize a face. Whole face, eyes, nose and mouth regions which could be used in a template matching strategy. The basis of the template matching strategy is to extract whole facial regions and compare these with the stored images of known individuals. Once again Euclidean distance can be used to find the closest match. For face recognition there are two types of comparisons are performed .The first is verification, in this the system compares the given individual with who that individual says they are and gives a yes or no decision. The second is identification. This is where the system compares the given individual to all the other individuals in the database [12]. All identification or authentication technologies operate using the following four stages: 
a. Capture: -Image is captured by the system

b. Extraction: - Unique data is extracted from the sample and a template is created.

c. Comparison: - The template is then compared with a new sample.

d. Match/non match: - The system decides if the features extracted from the new samples are a match or a not match.

First, skin detection approach based on novel skin color model is applied to check potential face regions. Detection of skin color in color images is a very popular and useful technique for face detection. While the input color image is typically in the RGB format, these techniques usually use color components in the color space, such as the HSV or YIQ formats. The skin pixel detection is performed with a simple color map, using the $\mathrm{YCbCr}$ color space. In the skin color detection process, each pixel was classified as skin or nonskin based on its color components [1]. First block also corresponds to the segmentation algorithm which is used to separate the image in the color filtered binary image into individual regions. The process consists of three steps. The first step is to fill up black isolated holes and to remove white isolated regions which are smaller than the minimum face area in training images. The highlighted region is converted into black lines and eroded to connect crossly separated pixels finally, the previous images are integrated into one binary image and relatively small black and white areas are removed.

After the segmentation, a set of connected homogenous skinlike regions is obtained. Once the set of FC is built, it is necessary to remove the ones that do not match to any face.

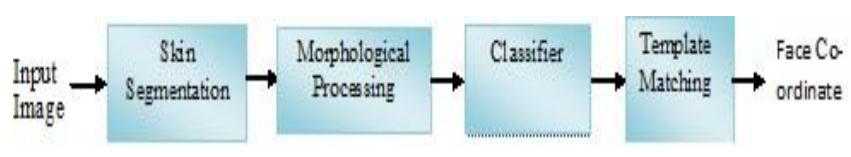

Fig.1 Steps of face detection

\subsubsection{Skin Segmentation}

The first step in the face detection algorithm is using skin segmentation to reject as much "non-face" of the image as possible, since the main part of the images consists of non-skin colour pixels. There are two ways of segmenting the image based on skin colour: Converting the RGB picture to $\mathrm{YCbCr}$ space or to HSV space. An YCbCr space segments the image into a luminosity component and colour components, whereas an HSV space divides the image into the three components of hue, saturation and colour value. The main advantage of converting the image to the $\mathrm{YCbCr}$ domain is that influence of luminosity can be removed during our image processing. It is important to note that the detection scheme should be invariant to skin type and changes in lighting condition therefore will make use of set of bounding rules for different colour space which will help to improve the detection efficiency[11][20].

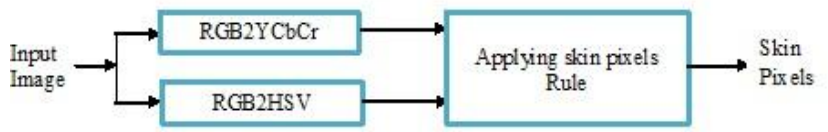

Fig. 2 Skin Segmentation

\subsubsection{Morphological Processing}

Skin colour segmentation will reject non skin colours from the input image but still the output image contain a bit of noise and clutter. For this reason series of morphological operations are performed to clean up the image. The goal is to end up with a mask image that can be applied to the input image to yield skin colour regions without noise and clutter.

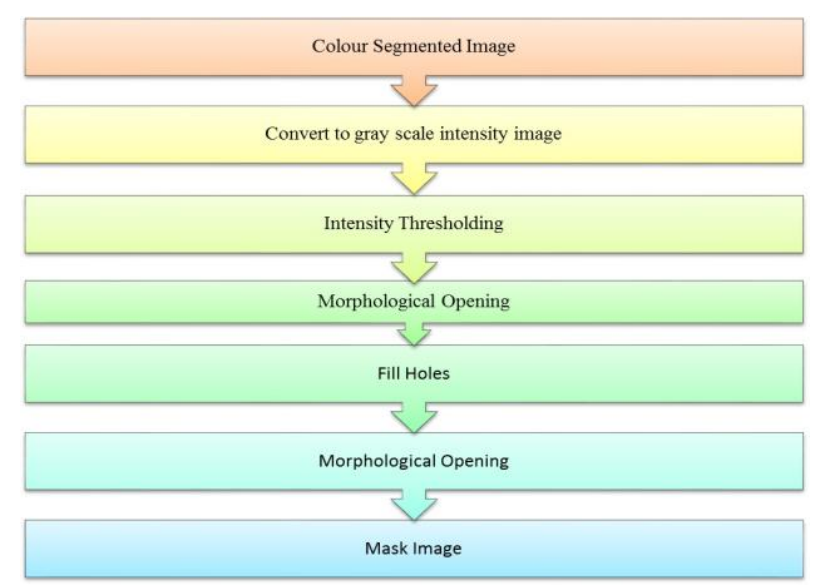

Fig. 3 Morphological Processing

Since morphological operations work on intensity images, the colour segmented image is converted into a gray scale image. Intensity thresholding is performed to break up dark regions into many smaller regions so that they can be cleaned up by morphological opening. The threshold is set low enough so that it doesn't clip away parts of a face but only create holes in it. Morphological opening is performed to remove very small objects from the image while preserving the shape and size of larger objects in the image [26].

\subsubsection{Template Matching}

In the template matching, we have used the trained images. We can easily find the position of the eyes, nose, mouth, eyebrows, head etc. by the use of template matching method. Therefore, we have detected the faces in this method.

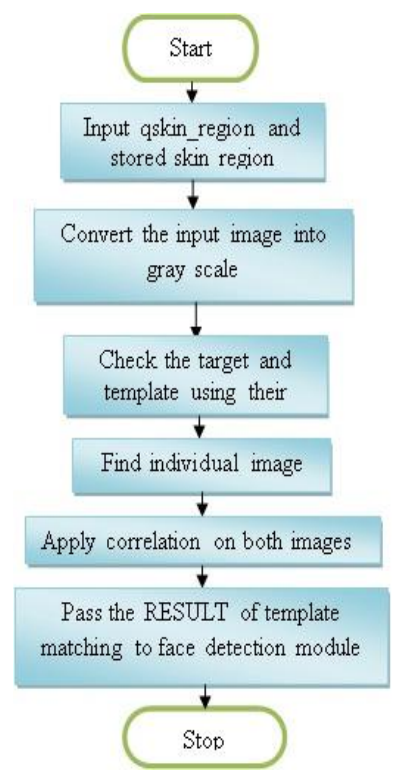

Fig.4 Template Matching Flow Graph 
The template was originally generated by cropping off all the faces in the training set using the ground truth data and averaging over them. It is observed that the intensity image obtained after colour segmentation contained faces with a neck region and so templates are modify to include the neck region. This was done by taking the intensity image after colour segmentation, separating out the connected regions, then manually selecting the faces and averaging over them [26].

\subsection{Eye and Mouth Detection}

Eye map means locating eyes based on feature maps derived from chrominance of an image [9]. The eye map from the chrominance is based on the observation that high $\mathrm{Cb}$ and low $\mathrm{Cr}$ values are found around the eyes. The steps involve in construction of eye map are as follow,

1. Convert the input image in $\mathrm{YCbCr}$ colour space.

2. Using the equation below eye maps based on chrominance component are built this is used further to detect the eyes [1].

3. Eye Map $\mathrm{C}=\frac{1}{3}\left\{\left(C_{b}^{2}\right)+\left(\tilde{C}_{r}\right)^{2}+\left(C_{b} / C_{r}\right)\right\}$ Where all components are normalized to the range [0; 255],

4. Perform dilation and erosion that is morphological operation on the image.

5. Finally eye map is constructed.

The colour of mouth region contains stronger red component and weaker blue component than other facial regions. Hence the chrominance component $\mathrm{Cr}$ is greater than $\mathrm{Cb}$ in the mouth region. The steps for the construction of mouth map are as follow,

1. Convert the input image in $\mathrm{YCbCr}$ colour space

2. We can construct the mouth map using formula,

$$
\text { Mouth Map }=C_{r}^{2} \cdot\left(C_{r}^{2}-\eta \cdot C_{r} / C_{b}\right)^{2}
$$

Where $\eta$ is,

$$
\eta=0.95 * \frac{(1 / n) \sum_{(x, y) \in F G} C_{r}(x, y)^{2}}{(1 / n) \sum_{(x, y) \in F G} C_{r}(x, y) / C_{b}(x, y)}
$$

Where both $C_{\mathrm{r}}^{2}$ and $C_{r} / C_{b}$ are normalized to the range [0; 255], and $\mathrm{n}$ is the number of pixels within the face mask, $F G$. The parameter $\eta$ is estimated as the ratio of the average $C_{r}^{2}$ to the average $C_{r} / C_{b}$.

1. Mouth map is dilated, masked, and normalized; it is dramatically brighter near the mouth areas than at other facial areas.

2. After all this processing mouth map is constructed.

\subsection{Yawning Detection}

A yawn is a reflex of simultaneous inhalation of air and stretching of the eardrums, followed by exhalation of breath. Yawning is commonly associated with tiredness, stress, overwork, lack of stimulation and boredom. There are two steps involved while detecting the yawn state. First of all yawn component in face independent of mouth location is detected. A criterion to detect the same is to find the hole in mouth as a result of wide mouth opening [1]. In second state mouth location is used to verify the correctness of detected component. The large hole is selected as a candidate for yawning mouth and this hole is the non-skin area inside the face. The hole can be related to eyes, mouth or open mouth. It is assumed that the open mouth will be the largest among three which can be a yawning state. In this way the detected yawn state is compared with the available face of yawning state to check the drowsiness of a driver. Figure shows procedure for computing yawning in the detected face. Input as detected face is taken for further processing. The mouth map and eye map of query image is calculated, then the size of query image mouth map is compared with the mouth map which are placed in existing database is checked. If size is found equal then the query image mouth map is compared with the mouth map from both the databases namely yawn face and normal face, after doing the comparison if mouth map match to any of the mouth map from the existing database then the respective message will be generated. The flowchart explains the process.

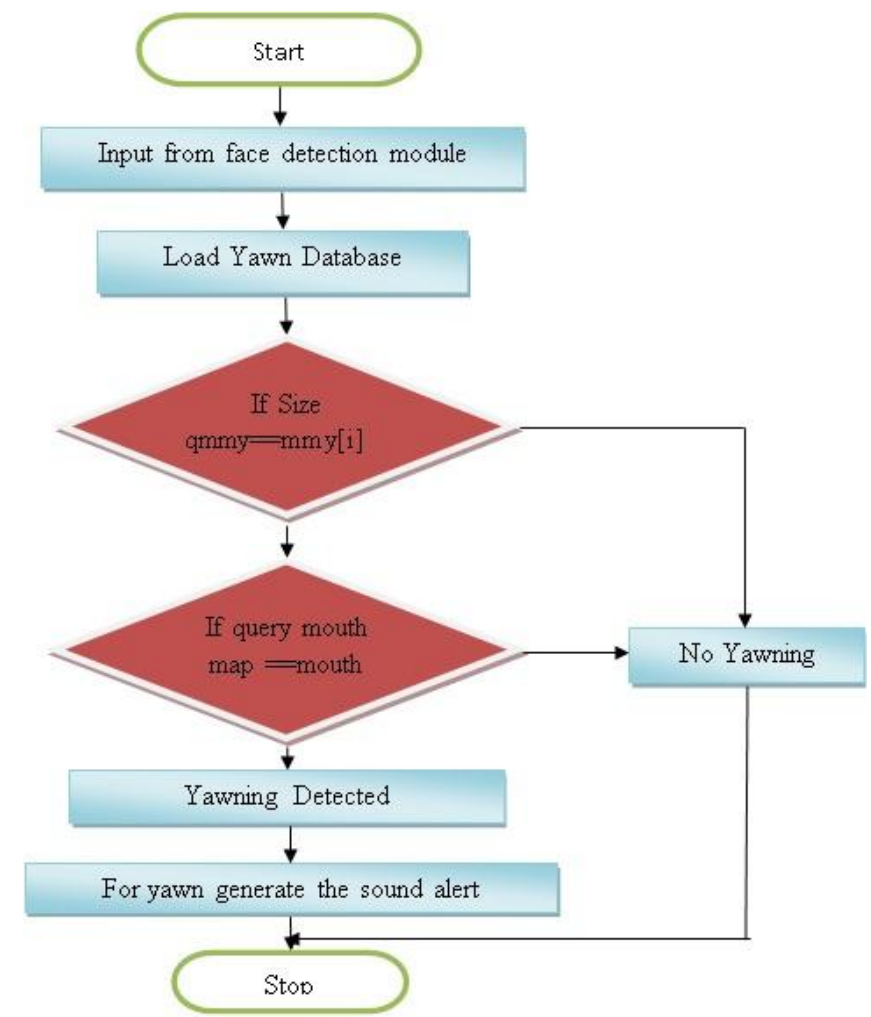

Fig. 5 Flowchart for Yawning Detection

The necessary steps to detect the yawn in image are:

1. Take the input image came from face detection module

2. Load the database of the yawn faces

3. Calculate the eye map and mouth map of query image

4. Compare the size of eye map and mouth map of query image with the maps stored in the database of yawn face.

5. If size matches then query image eye map and mouth map is compared with all the maps stored in the database.

6. If mouth map matches with mouth map in yawn face database then yawn is detected.

7. If yawn is found then sound alert is generated saying "Don't sleep you are driving".

8. If not yawn it means driver is not drowsy driver is driving safely. 


\section{APPLICATION}

Application of drowsiness detection systems will be primarily for driving on rural and other open highways, such as limitedaccess highways, at speeds at or above $50 \mathrm{mph}$. There are two reasons for limiting the drowsiness detection system to this domain. First, most drowsiness-related crashes occur on these roads at these speeds. Second, it appears that this domain is the one in which probability is maximized. Some other usefulness of drowsiness detection system comes under the area of machine learning techniques which can automate the analysis of data providing a much more rapid cycle of hypothesizing and experimentation. Having an automated system allows hypotheses to be tested in a much higher bandwidth fashion. Develop a low cost system to identify feasible indicators of driver sleepiness and distraction. Implement and validate camera-based indicators of driver sleepiness, investigate the performance of the indicators as sleepiness detectors.

\section{EXPERIMENTAL RESULT}

\subsection{Detection GUI}

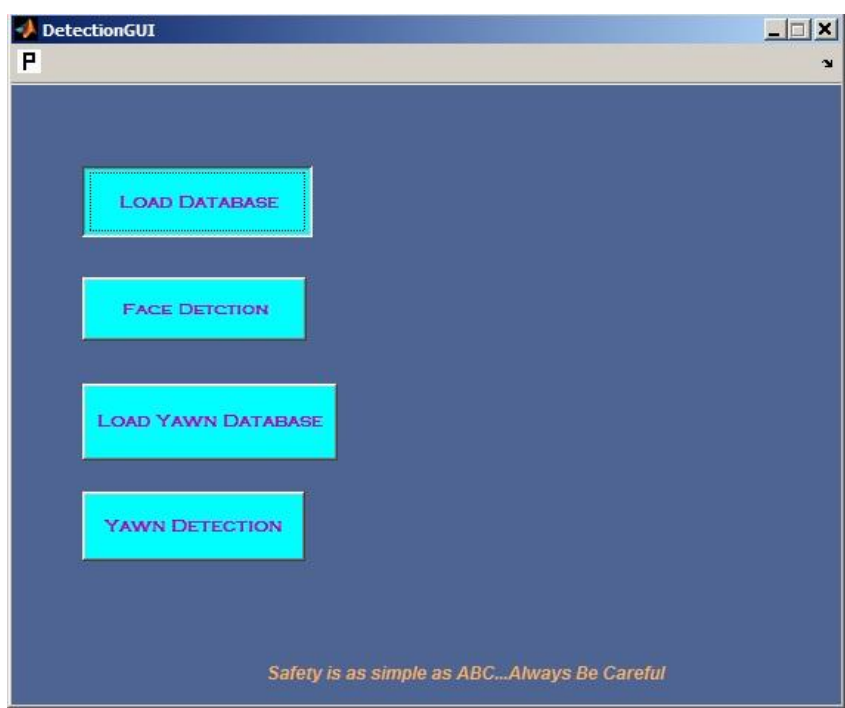

\subsection{Face Detection Module}

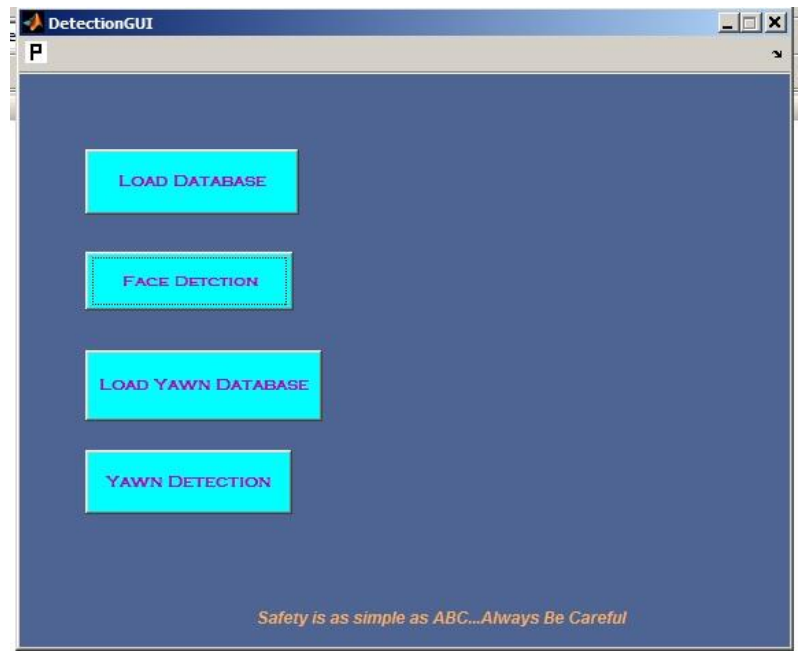

\subsection{Face Detected}

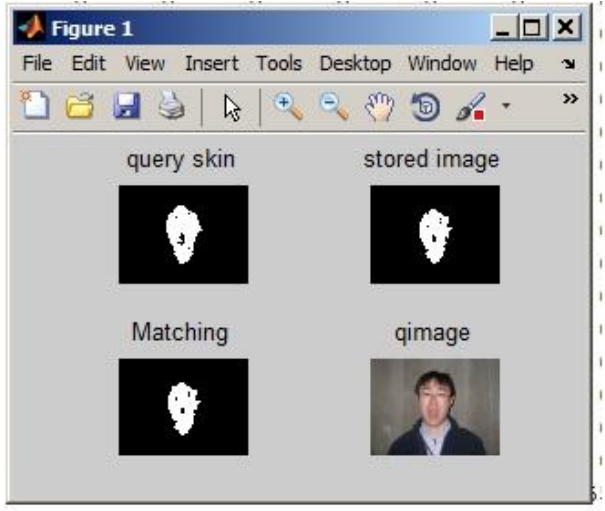

face detected

\subsection{Load Yawning detection Database}

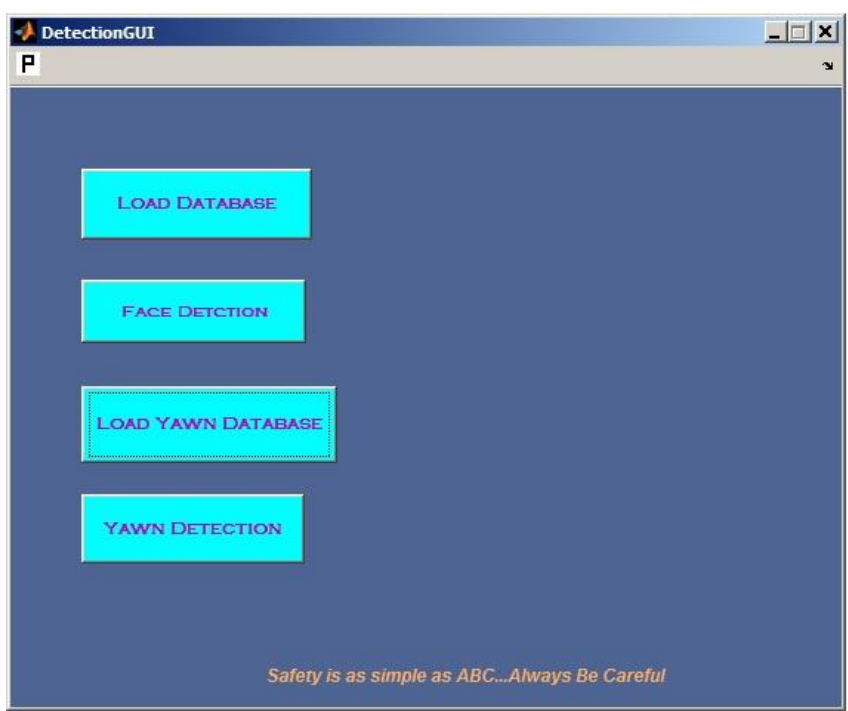

\subsection{Yawn Detected and Alert Generated}

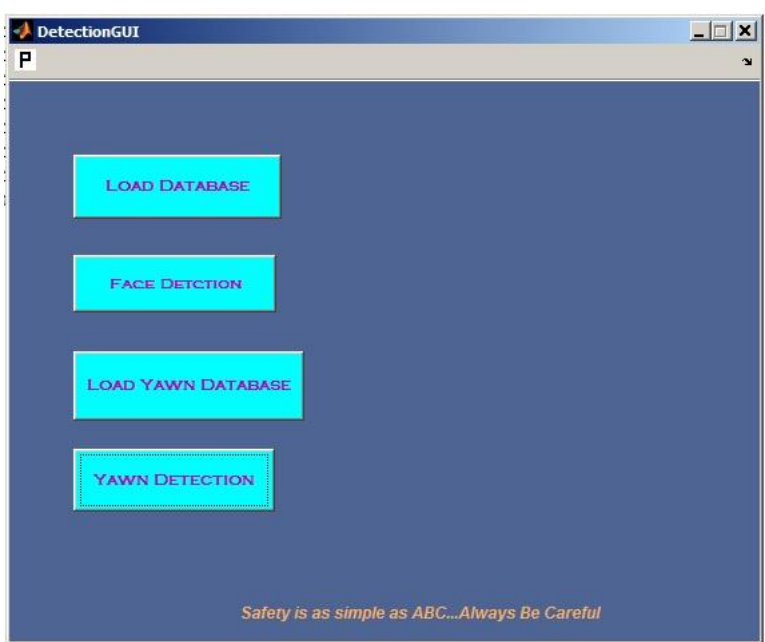




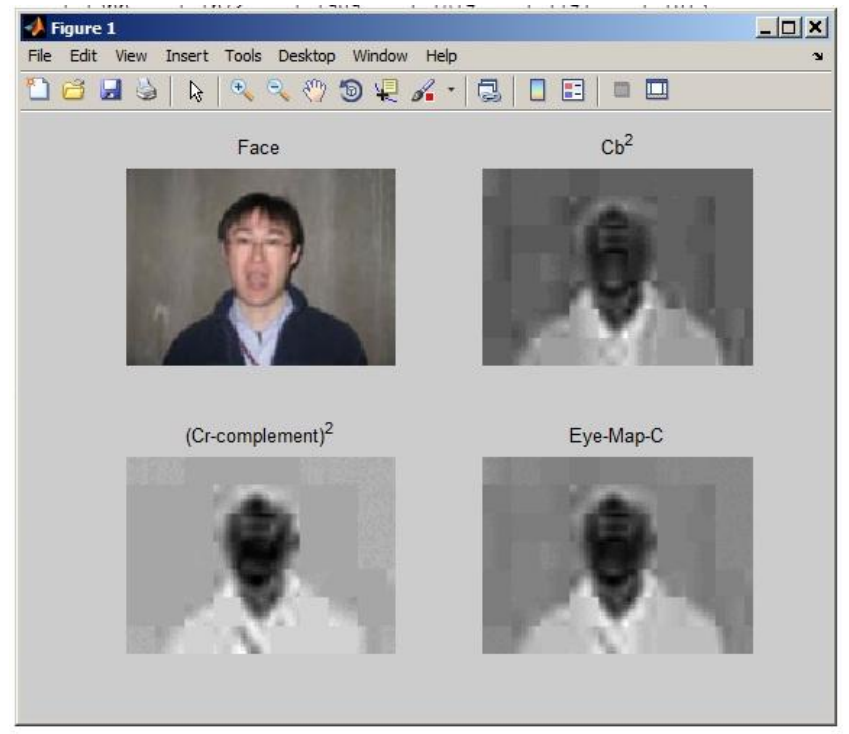

yawn detected

\section{Sound alert arrives "Don't sleep you are driving"}

\section{CONCLUSION}

In this proposed system method for detecting driver yawning is implemented, where face is detected after that calculation of the eye map and mouth map will be performed. For any input image it will check the yawn by comparing the mouth map of query image with the stored image mouth map in database. For doing this it is necessary to store the yawn face and normal face in database which one has to give as input. So if the mouth map matches using yawn face it will generate the sound alert "'Don't sleep you are driving" otherwise no alert for no yawn.

The aim to build this system is to provide a product which will assist driver while driving such that though driver is drowsy we can alert the driver at earlier stage to avoid further mishaps. Image processing achieves highly accurate and reliable detection of drowsiness. Image processing offers a non-invasive approach to detecting drowsiness without the annoyance and interference. A drowsiness detection system developed around the principle of image processing judges the driver's alertness level on the basis of yawning behaviour.

\section{FUTURE SCOPE}

As we know there is always a scope for further improvement same thing will be applicable over here. There is vast scope for this system of drowsiness detection. As there are several signs by which we can say the person is feeling drowsy. To detect the drowsiness several alternatives are available like eye detection, Iris detection, pupil detection, and yawing detection. Out of these options proposed system uses yawning behaviour for drowsiness detection. For making the system more strong and efficient one can check the two different behaviour together so chances of false identification may reduce. This system can be introduced in an organization like security system, Toll collection counters, importantly at check post.

\section{REFERENCES}

[1] Shabnam Abtahi,Behnoosh,Driver Monitoring Based on Yawning Distributed Collaborative Virtual

Drowsiness Detection, Environment
[2] S.G.Klauer, T.A Dingus,Neale, V.L.,Sudweeks, J.D., and Ramsey, DJ, "The Impact of Driver Inattentation on Near-Crash/Crash Risk: An Analysis Using the 100-Car Neutralistic Driving Study Data,"Virginia Tech Transportation Instittute Report \#DOT HS 810594

[3] U. Yufeng, W. Zengcai, "Detecting Driver Yawning in Successive images."In: Proc. Sixth International Conf. on Bioinformatics and Biomedical Engineering, 2007, pp.581-583

[4] X. Fan, B. Yin, Y. Fun. "Yawning Detection for Monitoring Driver Fatigue." In: Sixth International Conf. on Machine Learning and Cyernetics, Hong Kong, 2007, pp. 664-668

[5] T.Azim, M.A Jaffer, A.M Mirza. Äutomatic Fatigue Detection of Drivers through Pupil Detection and Yawning Analysis.'In:Proc. Fourth International Conf. on Innovative Computing, Information and Control, 2009, pp. 441-445

[6] L. Li, Y. Chen , Z. Li. "Yawning Detection for Monitoring Driver Fatigue based on Two Camera."In:Proc. $12^{\text {th }}$ International IEEE conf. on Intelligent Transportation Systems, St. Louis, MO, USA, 2009, pp.12-17

[7] T.Wang, P. Shi. "Yawning Detection for Determining Driver Drowsiness."IEE International Workshop VL.SI Design \& Video Tech. Suzhou China 2005 page 373-376.

[8] M.H Yang, D.J Kriegman, and N.Ahuja "Detecting faces in images: A Survey."'IEE Trans. Pattern Analysis and Machine Intelligence, Vol.24, No. 1, pp. 34-58, 2002

[9] N. A. A.Rahman, K.C. Wei and J.See. "RGB-H$\mathrm{CBCr}$ Skin Colour Model for Human Face Detection" In Proceedings of The MMU International Symposium on Information \& Communication s Technologies, 2006

[10] Hsu Rein-Lien, M. Abdel-Mottaleb, and. A. K. Jain. "Face Detection in color Images." IEEE Trans. Pattern Analysis and Machine Intelligence, Vol.24, issue 5. 2002

[11] Rein-Lien Hsu,2002, Face Detection and Modelling for Recognition

[12] Australian Transport Safety Bureau, "Road safety research report OR23. Fatigue related crashes: An analysis of fatigue-related crashes on Australian roads using an operational definition of fatigue."

[16] Xiao Fan, Bao-Cai Yin, Yan-Feng Sun "Yawning Detection For Monitoring Driver Fatigue “

[17] Drowsy driving and automobile crashes, report and recommendation, National centre on sleep disorders research, National Highway Traffic Safety Administration

[18] Yihu Yi 1,2 Daokui Qu 1,3 Fang Xu 1,3,Face detection method based on skin color segmentation and eyes Verification, 
[19] Inseong Kim, Joon Hyung Shim, and Jinkyu Yang ,Face detection

[20] Ana Bertran, Huanzhou Yu, Paolo Sacchetto, Face Detection Project Report

[21] Hypo vigilance Warning System: A Review on Driver Alerting Techniques,S Arun, M Murugappan, Kenneth Sundaraj, 2011 IEEE Control and System Graduate Research Colloquium

[23] Deepesh Jain, Husrev Tolga Ilhan,Subbu Meiyappan, Face Detection using Template Matching, EE 368 Digital Image Processing Spring 2002-2003

[24] Smita Tripathi,SOIT, RGPV Bhopal,Varsha Sharma SOIT, RGPV Bhopal,Sanjeev Sharma, SOIT ,Face Detection using Combined Skin Color Detector and Template Matching Method, RGPV Bhopal International Journal of Computer Applications (0975 - 8887) Volume 26- No.7, July 2011
[25] Waqar Mohsin,Noman Ahmed, Chung-Tse Mar May 26, 2003 EE368 Digital Image Processing, Spring 2002-2003 Department of Electrical Engineering,Stanford University , "Face Detection Project"

[26] Srikanth Rangarajan, "Algorithms for edge detection"

[27] nurul asmira bt ahmad agin, Eye detection , November 2009 